\title{
An elastic-viscous-plastic structured UH model
}

\author{
Zhu Enyang ${ }^{1 *}$, Wang Yihe ${ }^{1}$ \\ ${ }^{1}$ North China University of Technology, School of Civil Engineering, Beijing, 100144, China
}

\begin{abstract}
On the basis of the structured unified hardening model (structured UH model) considering soil structured collapse, the time effect is take into account, and an elastic-viscous-plastic structured UH model is extended. In the presented model, the moving normal compression line (MNCL) is extended to a moving instant normal compression line (MINCL) for simulating both soil structure effect and time effect. Then the instance plastic strains irrespective of time effect are calculated by referring the MINCL, and the plastic strains due to time effect are calculated by referring the traditional instant normal compression line. Comparisons between test data and model simulation indicate that the presented elastic-viscous-plastic structured UH model is qualified to simulate the combined actions of soil structure and time effects both in compression and in shearing.
\end{abstract}

\section{Introduction}

Soil structure is widely existed in natural soils, which leads two main effects on the mechanical properties: (1) existence of soil structure leads greater strength than the corresponding reconstituted soils. (2) Decay of soil structure cause greater volumetric deformation than the corresponding reconstituted soils. Thus structured soils and reconstituted ones behave very different in stress-strain relationships. On the other hand, the stress-strain relationships of soils are usually considered to be time-dependent, especially for soft clays. Therefore, in constitutive model expression, it is necessary to take into account the influence of both the soil structure and the time dependence.

Aiming at soil structure and time dependence, many scholars presented a lot of outstanding achievements. According to the microscopic deformation mechanism of natural clay, the deformation of structured soil is divided into three phases as elastic, structural strength damage and rebounded soil, and an elasto-viscoplastic damage model was developed ${ }^{1}$. Based on a masonry model, a viscous-elastic-plastic model for structured clays was proposed by using overstress theory ${ }^{2}$. Based on experimental observations, a strain-rate based model is developed to describe the mechanical behavior for both unstructured and structured clays under one-dimensional loading ${ }^{34}$. Taking into account the non-linear feature of semi-logarithmic compression curves for natural clays as well as the variation behavior of creep rate related to the stress level and consolidation duration time, a modified elastic visco-plastic model was proposed ${ }^{5}$. After proposing a new concept of viscoplastic strain rate lines and a new description for the progressive destruction during one-dimentional loading on structured clays, a one-dimentional elastic visco-plastic model considering destruction effects was established ${ }^{6}$. By introducing a moving normal compression line (MNCL), the unified hardening model(UH model) for reconstituted soil was extended to a structured UH model mainly considering soil structure collapse ${ }^{7}$. In this paper, the structured UH model is then attempted to be extended to an elastic-viscous-plastic structured UH model (EVP structured UH model).

\section{EVP structured UH model}

\subsection{Review of the structured UH model}

The current and reference yield surfaces $f$ and $\bar{f}$ in meridian space are shown in Figure 1.

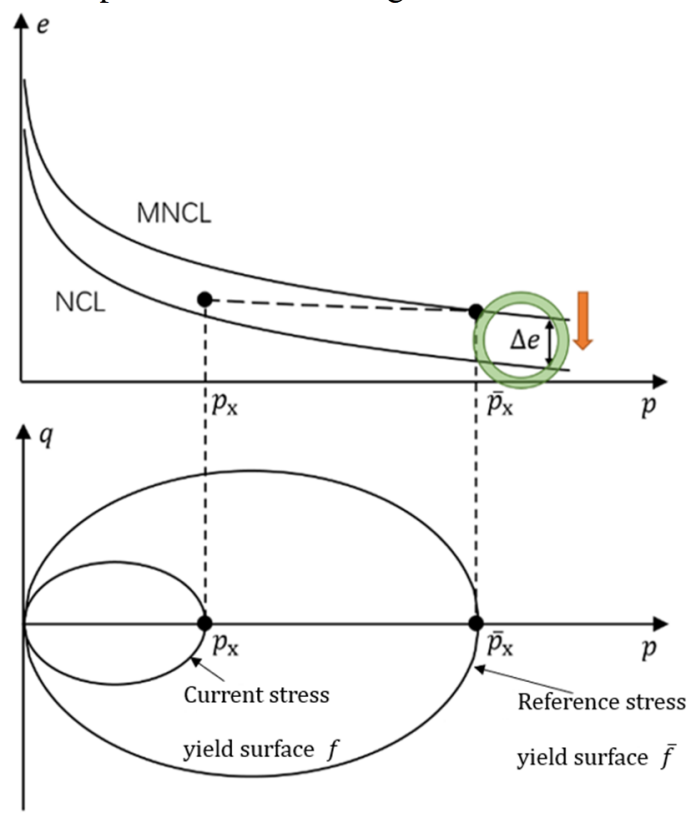

Figure 1. Yield surfaces of structured UH model

Corresponding author: zhuenyang@ncut.edu.cn 


$$
\left.\begin{array}{l}
f=p\left(1+\frac{\eta^{2}}{M^{2}}\right)-p_{\mathrm{x}}=0 \\
\bar{f}=\bar{p}\left(1+\frac{\bar{\eta}^{2}}{M^{2}}\right)-\bar{p}_{\mathrm{x}}=0
\end{array}\right\}
$$

In equation (1): stress ratio $\eta=q / p, \bar{\eta}=\bar{q} / \bar{p} ; p$ represents mean principal stress; $q$ represents generalized shear stress; $(p, q)$ and $(\bar{p}, \bar{q})$ respectively represent the stress state on current and reference yield surface; $p_{\mathrm{x}}$ and $\bar{p}_{\mathrm{x}}$ are respectively the intercepts of the two yield surfaces on $p$ axis. The evolutions of $p_{\mathrm{x}}$ and $\bar{p}_{\mathrm{x}}$ are shown in equation (2):

$$
\left.\begin{array}{c}
p_{\mathrm{x}}=p_{\mathrm{x} 0} \exp \left(\frac{1}{c_{\mathrm{p}}} \int \mathrm{d} H\right) \\
\bar{p}_{\mathrm{x}}=\bar{p}_{\mathrm{x} 0} \exp \left[\frac{1}{c_{\mathrm{p}}} \int \mathrm{d} \varepsilon_{\mathrm{v}}^{\mathrm{p}}+\frac{1}{\lambda-\kappa} \int \mathrm{d}(\Delta e)\right]
\end{array}\right\}
$$

In equation (2): $c_{\mathrm{p}}=(\lambda-\kappa) /\left(1+e_{0}\right) ; \lambda$ and $\kappa$ are respectively the slops of isotropic compression and swelling lines of normal compression soil; $e_{0}$ is the initial void ratio; $p_{\mathrm{x} 0}$ and $\bar{p}_{\mathrm{x} 0}$ are respectively the initial values of $p_{\mathrm{x}}$ and $\bar{p}_{\mathrm{x}} ; \int \mathrm{d} H$ is the hardening parameter:

$$
\int \mathrm{d} H=\int R^{\frac{\Delta e}{\Delta e_{0}}\left(\eta-M_{\mathrm{f}}\right)} \frac{M_{\mathrm{f}}^{4}-\eta^{4}}{M^{4}-\eta^{4}} \mathrm{~d} \varepsilon_{\mathrm{v}}^{\mathrm{p}}
$$

In equation (3): $M$ is the critical state stress ratio; $\Delta e$ is the current soil structure potential; $\Delta e_{0}$ is the initial value of $\Delta e ; M_{\mathrm{f}}$ is the potential failure stress ratio:

$$
M_{\mathrm{f}}=6\left[\sqrt{\frac{\chi}{R}\left(1+\frac{\chi}{R}\right)}-\frac{\chi}{R}\right]
$$

In equation (4): $\chi=M^{2} /[12(3-M)] ; R=p_{\mathrm{x}} / \bar{p}_{\mathrm{x}}$;

$$
R=\frac{p_{\mathrm{X}}}{\overline{\bar{p}_{\mathrm{x}}}}=\frac{p\left(1+\frac{\eta^{2}}{M^{2}}\right)}{\bar{p}_{x 0}} \exp \left[-\frac{1}{c_{\mathrm{p}}} \int \mathrm{d} \varepsilon_{\mathrm{v}}^{\mathrm{p}}-\frac{1}{\lambda-\kappa} \int \mathrm{d}(\Delta e)\right]
$$

In equation (5): the increment $\mathrm{d}(\Delta e)$ is expressed as:

$$
\mathrm{d}(\Delta e)=-\zeta R \Delta e\left\langle R^{\frac{\Delta e}{\Delta e_{0}}\left(\eta-M_{\mathrm{f}}\right)} \frac{M_{\mathrm{f}}^{4}-\eta^{4}}{M^{4}-\eta^{4}} \mathrm{~d} \varepsilon_{\mathrm{v}}^{\mathrm{p}}\right\rangle
$$

In equation (6): $\zeta$ is soil structure decay rate; the Macaulay parenthesis " \langle\rangle " is expressed as:

$$
\langle x\rangle= \begin{cases}x & x \geq 0 \\ 0 & x<0\end{cases}
$$

According to the associative flow rule, the plastic potential function $g=f$ is expressed as:

$$
g=f=p\left(1+\frac{\eta^{2}}{M^{2}}\right)-p_{\mathrm{x}}=0
$$

According to equations (1)-(8), the plastic volumetric and generalized shear strain increments $\mathrm{d} \varepsilon_{\mathrm{v}}^{\mathrm{p}}$ and $\mathrm{d} \varepsilon_{\mathrm{d}}^{\mathrm{p}}$ for the structured UH model are expressed as:

$$
\left.\begin{array}{c}
\mathrm{d} \varepsilon_{\mathrm{v}}^{\mathrm{p}}=c_{\mathrm{p}} R^{\frac{\Delta e}{\Delta e_{0}}\left(M_{\mathrm{f}}-\eta\right)} \frac{M^{4}-\eta^{4}}{M_{\mathrm{f}}^{4}-\eta^{4}} \frac{\mathrm{d} p_{\mathrm{x}}}{p_{\mathrm{x}}} \\
\mathrm{d} \varepsilon_{\mathrm{d}}^{\mathrm{p}}=c_{\mathrm{p}} R^{\frac{\Delta e}{\Delta e_{0}}\left(M_{\mathrm{f}}-\eta\right)} \frac{M^{4}-\eta^{4}}{M_{\mathrm{f}}^{4}-\eta^{4}} \frac{2 \eta}{M^{2}-\eta^{2}} \frac{\mathrm{d} p_{\mathrm{x}}}{p_{\mathrm{x}}}
\end{array}\right\}
$$

\subsection{EVP structured UH model}

Obviously, strains due to time effects is generally considered as unrecoverable, thus in EVP constitutive models, the strain $\varepsilon_{i j}$ is divided into three parts: $\varepsilon_{i j}^{\mathrm{e}}$ for recoverable elastic strain; $\varepsilon_{i j}^{\mathrm{sp}}$ for unrecoverable plastic strain due to stress; and $\varepsilon_{i j}^{\text {tp }}$ for unrecoverable plastic strain due to time effect.

$$
\varepsilon_{i j}=\varepsilon_{i j}^{\mathrm{e}}+\varepsilon_{i j}^{\mathrm{sp}}+\varepsilon_{i j}^{\mathrm{tp}}
$$

According to the literature, an instant normal compression line (INCL) was introduced to reflect instantaneous isotropic compression behaviors of normally consolidated soil. Applying INCL instead of NCL as the reference line to determine the internal variable $R$, time effect on the stress-strain relationship for reconstituted soil can be considered ${ }^{8}$.

In order to reflect both soil structure and time effect, the INCL is extended to be a moving INCL (MINCL), which parallels to the INCL and moves downward with soil structure decay. Thus the yield surfaces are the same as that indicated in equation (1), while the evolutions of $p_{\mathrm{x}}$ and $\bar{p}_{\mathrm{x}}$ are revised as:

$$
\left.\begin{array}{c}
p_{\mathrm{x}}=p_{\mathrm{x} 0} \exp \left(\frac{1}{c_{\mathrm{p}}} \int \mathrm{d} H-\bar{t}\right) \\
\bar{p}_{\mathrm{x}}=\bar{p}_{\mathrm{x} 0} \exp \left[\frac{1}{c_{\mathrm{p}}} \int\left(\mathrm{d} \varepsilon_{\mathrm{v}}^{\mathrm{sp}}+\mathrm{d} \varepsilon_{\mathrm{v}}^{\mathrm{tp}}\right)+\frac{1}{\lambda-\kappa} \int \mathrm{d}(\Delta e)\right]
\end{array}\right\}
$$

In equation (11), hardening parameter $H$ is revised as:

$$
H=\int R^{\frac{\Delta e}{\Delta e_{0}}\left(\eta-M_{\mathrm{f}}\right)} \frac{M_{\mathrm{f}}^{4}-\eta^{4}}{M^{4}-\eta^{4}}\left(\mathrm{~d} \varepsilon_{\mathrm{v}}^{\mathrm{sp}}+\mathrm{d} \varepsilon_{\mathrm{v}}^{\mathrm{tp}}\right)=\int \frac{1}{\Omega} \mathrm{d} \varepsilon_{\mathrm{v}}^{\mathrm{p}}
$$

And the conversion time $\bar{t}$ is expressed as:

$$
\left.\begin{array}{c}
\bar{t}=\frac{C_{\alpha \mathrm{e}}}{\lambda-\kappa} \int \frac{M_{\mathrm{f}}^{4}}{M^{4}} R_{\mathrm{t}}^{\alpha} \mathrm{d} t \\
\alpha=(\lambda-\kappa) / C_{\alpha \mathrm{e}}
\end{array}\right\}
$$

In equation (13): $C_{\alpha \mathrm{e}}$ is the secondary consolidated coefficient; internal variable $R_{\mathrm{t}}$ is expressed as:

$$
\left.\begin{array}{c}
R_{\mathrm{t}}=R_{0} \frac{\exp \left(\frac{1}{c_{\mathrm{p}}} \int \frac{M_{\mathrm{ft}}^{4} \eta^{4}}{M^{4}-\eta^{4}} \mathrm{~d} \varepsilon_{\mathrm{v}}^{\mathrm{sp}}\right)}{\exp \left(\frac{1}{c_{\mathrm{p}}} \int \mathrm{d} \varepsilon_{\mathrm{v}}^{\mathrm{p}}\right)} \\
M_{\mathrm{ft}}=6\left[\sqrt{\frac{\chi}{R_{\mathrm{t}}}\left(1+\frac{\chi}{R_{\mathrm{t}}}\right)}-\frac{\chi}{R_{\mathrm{t}}}\right]
\end{array}\right\}
$$

The elastic volumetric strain increment $\mathrm{d} \varepsilon_{\mathrm{v}}^{\mathrm{e}}$ and elastic generalized shear strain increment $\mathrm{d} \varepsilon_{\mathrm{d}}^{\mathrm{e}}$ are respectively calculated by Hooke's law:

$$
\left.\begin{array}{c}
\mathrm{d} \varepsilon_{\mathrm{v}}^{\mathrm{e}}=\frac{\kappa}{1+e_{0}} \frac{\mathrm{d} p}{p}=\frac{\mathrm{d} p}{K} \\
\mathrm{~d} \varepsilon_{\mathrm{d}}^{\mathrm{e}}=\frac{2(1+v) \kappa}{9\left(1+e_{0}\right)(1-2 v)} \frac{\mathrm{d} q}{p}=\frac{\mathrm{d} q}{3 G}
\end{array}\right\}
$$

In equation (15): $v$ is Poisson's ratio.

According to equations (1), (4), (5), (6), (8), and (11)-(15), the elastic-viscous-plastic stress-strain relationship for structured soil can be expressed as:

$$
\left[\begin{array}{l}
\mathrm{d} \varepsilon_{\mathrm{v}} \\
\mathrm{d} \varepsilon_{\mathrm{d}}
\end{array}\right]=\left[\begin{array}{ll}
A & B \\
C & D
\end{array}\right]\left[\begin{array}{l}
\mathrm{d} p-B_{1} \mathrm{~d} t \\
\mathrm{~d} q-B_{2} \mathrm{~d} t
\end{array}\right]
$$

In equation (16): $A=c_{\mathrm{p}} \Omega \frac{\partial f}{\partial p}+\frac{1}{K} ; B=c_{\mathrm{p}} \Omega \frac{\partial f}{\partial q}$;

$C=c_{\mathrm{p}} \Omega \frac{\partial f}{\partial p} \frac{2 \eta}{M^{2}-\eta^{2}} ; D=c_{\mathrm{p}} \Omega \frac{\partial f}{\partial q} \frac{2 \eta}{M^{2}-\eta^{2}}+\frac{1}{3 G} ;$

$B_{1}=\frac{p c_{\mathrm{p}} K \frac{C_{\alpha \mathrm{e}} M_{\mathrm{f}}^{4}}{\lambda-\kappa M^{4}} R_{\mathrm{t}}^{\alpha}\left(M^{4}-\eta^{4}\right)}{c_{\mathrm{p}} K\left(M^{2}-\eta^{2}\right)^{2}+12 c_{\mathrm{p}} \eta^{2} G+p\left(M_{\mathrm{f}}^{4}-\eta^{4}\right)} ;$

$B_{2}=\frac{6 q c_{\mathrm{p}} G \frac{C_{\alpha \mathrm{e}} M_{\mathrm{f}}^{4}}{\lambda-\kappa M^{4}} R_{\mathrm{t}}{ }^{\alpha}\left(M^{2}+\eta^{2}\right)}{c_{\mathrm{p}} K\left(M^{2}-\eta^{2}\right)^{2}+12 c_{\mathrm{p}} \eta^{2} G+p\left(M_{\mathrm{f}}^{4}-\eta^{4}\right)}$

There are in all 8 parameters in the presented model: $\lambda, \kappa, M, v, \Delta e_{0}, \zeta, C_{\alpha \mathrm{e}}$, and value of void ratio $N_{\mathrm{i}}$ for $p=1 \mathrm{kPa}$ on the traditional INCL. All the model parameters can be determined by in-situ tests on the structured soils and the corresponding reconstituted soils according to the literatures 7 and 8 . 


\section{Model Evolutions}

In this section, the presented elastic-viscous-plastic structured UH model is indicated in isotropic compression and undrained shear simulations. The model parameters used for the simulations are shown in Table1.

As shown in Figure 2, for isotropic compression, with the decrease of loading rate $\dot{\varepsilon}_{\mathrm{V}}$, compression yielding stress decreases. And compression lines of different loading rates finally tend to be parallel to each other.

Table1. Model parameters used for simulations

\begin{tabular}{cccccc}
\hline$\lambda$ & $\kappa$ & $N_{\mathrm{i}}$ & $M$ & $v$ & $C_{\alpha \mathrm{e}}$ \\
\hline 0.2 & 0.03 & 2.0 & 1.5 & 0.25 & 0.05 \\
\hline
\end{tabular}

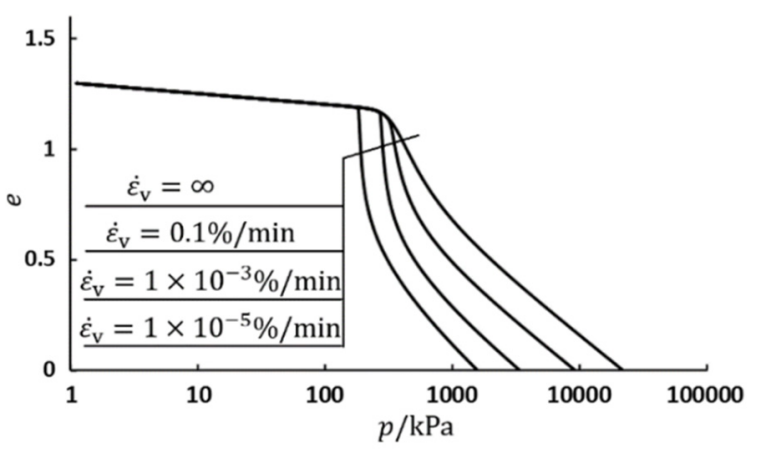

Figure 2. Effect of loading rate on isotropic compression

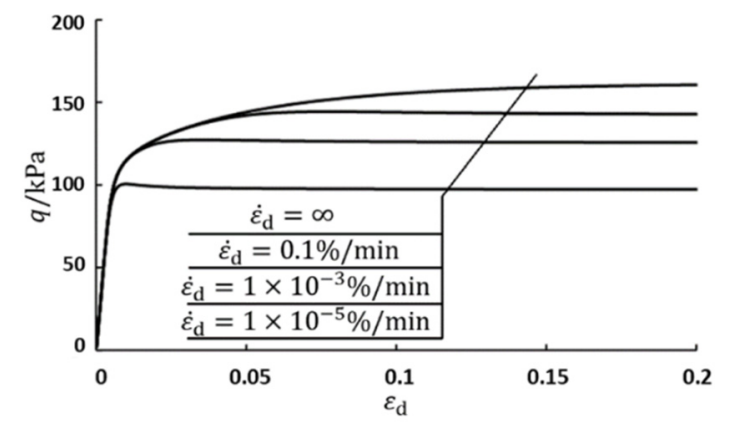

Figure 3. Effect of loading rate on undrained shear

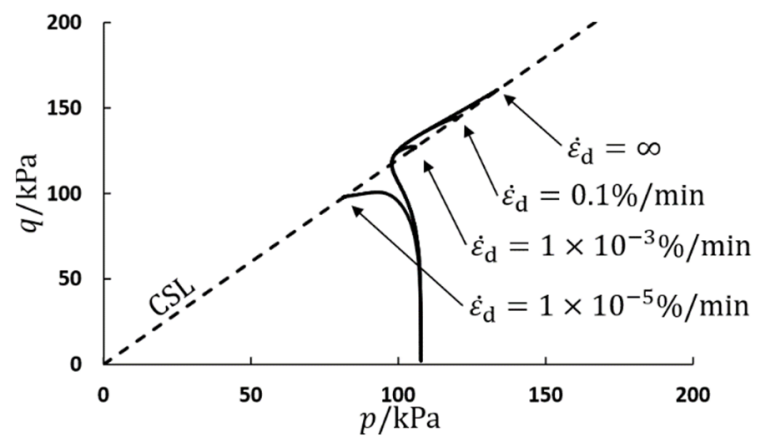

Figure 4. Stress paths of undrained shear in different loading rate

As shown in Figure 3 and 4, in undrained shear simulations, with the decrease of shear strain rate $\dot{\varepsilon}_{\mathrm{d}}$, the shear strengths decrease.

\section{Model prediction}

In order to verify the reasonability of the presented elastic-viscous-plastic structured UH model, experimental data of 4 structured soils are compared with the corresponding model predictions in this part. The model parameters used are shown in Table 2.

Table 2. Model parameters of 4 natural clays

\begin{tabular}{ccccc}
\hline & Ariake & Batiscan & Berthierville & Osaka \\
\hline$\lambda$ & 0.27 & 0.39 & 1.2 & 0.194 \\
$\kappa$ & 0.016 & 0.037 & 0.05 & 0.015 \\
$N_{\mathrm{i}}$ & 3.48 & 3.56 & 6.52 & 2.43 \\
$M$ & 1.5 & 1.3 & 1.2 & 1.59 \\
$\nu$ & 0.25 & 0.25 & 0.1 & 0.25 \\
$C_{\alpha \mathrm{e}}$ & 0.05 & 0.06 & 0.08 & 0.0059 \\
$\Delta e_{0}$ & 0.67 & 0.61 & 1.7 & 0.52 \\
$\zeta$ & 10.0 & 80.0 & 15.0 & 22.0 \\
\hline
\end{tabular}

Undisturbed Ariake clay ${ }^{9}$ was sampled from $1 \mathrm{~m}$ to $21 \mathrm{~m}$ below earth surface in Saga Plain. Then the samples were implemented oedometer test in constant rate of strain (CRS tests). The test data and model predictions are shown in Figure 5. Batiscan situated on the north shore of the St Lawrence River, about $110 \mathrm{~km}$ west of Quebec City. The clay tested was taken at a depth of $7.25-7.46 \mathrm{~m}$, whose pre-consolidation pressure is $88 \mathrm{kPa}^{10}$. The CRS test data and model predictions for Batiscan clay are shown in Figure 6. Berthierville located on the north shore of the Saint Laurent River between Québec City and Montréal ${ }^{11}$. The CRS test data and model predictions for Berthierville clay are shown in Figure 7. According to Figure 5 to 7 , it is not difficult to find that the proposed model is qualified in a simulating the compression behavior of structured soil considering time effect.

Osaka located in the Tsurumi area east of Osaka, Japan. The Osaka clay ${ }^{12}$ specimens were taken from $8.3 \mathrm{~m}$ below earth surface and their void ratios ranged from 1.7 to 1.9. Figure 8 and 9 show the predictions of undrained shearing on Osaka clay, and the loading rates are listed in Table 3. Though the prediction of undrained shear test is not as good as that of the compression, the predictions approximately simulate both stress-strain relationship and effective stress path, and both of strain hardening and strain softening with positive pore water pressure can also be described consistently.

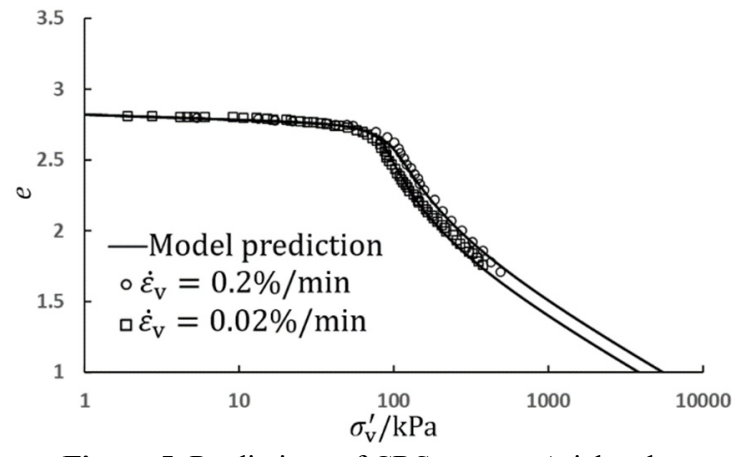

Figure 5. Predictions of CRS tests on Ariake clay 


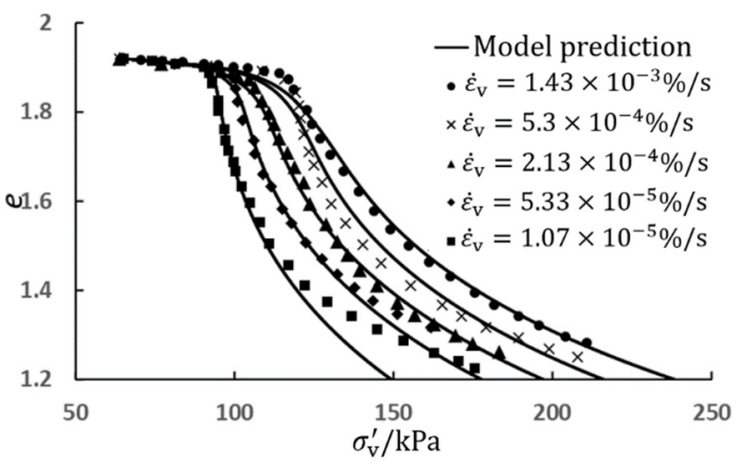

Figure 6. Predictions of CRS tests on Batiscan clay

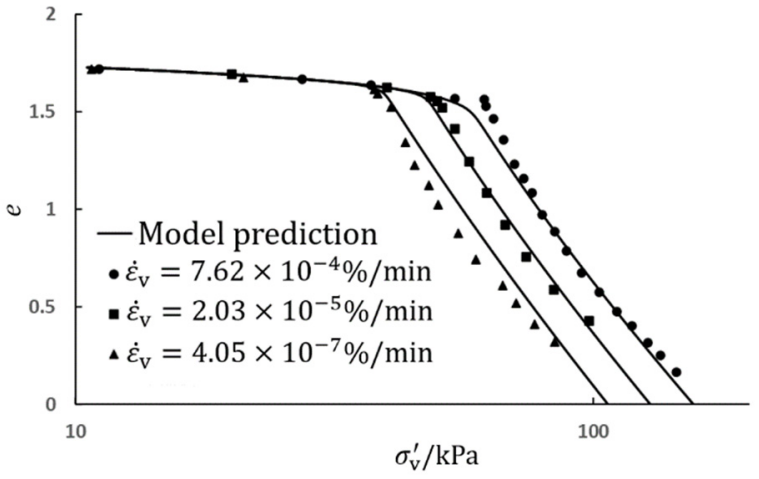

Figure 7. Prediction of CRS tests on Berthierville clay

Table 3. The conditions a of the Osaka clay specimens

\begin{tabular}{cccc}
\hline Specimen & $\begin{array}{c}\text { Consolidation } \\
\text { pressure }(\mathrm{kPa})\end{array}$ & $\begin{array}{c}\text { Loading rate } \\
(\% / \mathrm{min})\end{array}$ & $\begin{array}{c}\text { Initial void } \\
\text { ratio }\end{array}$ \\
\hline TSK-2 & 58.8 & $1.00 \times 10^{-2}$ & 1.91 \\
TSK-5 & 39.2 & $5.48 \times 10^{-4}$ & 1.92 \\
TSK-6 & 19.6 & $1.00 \times 10^{-2}$ & 1.90 \\
TSK-7 & 117.6 & $5.48 \times 10^{-4}$ & 1.92 \\
TSK-8 & 176.4 & $1.00 \times 10^{-2}$ & 1.91 \\
TSK-9 & 235.2 & $1.00 \times 10^{-2}$ & 1.91 \\
\hline
\end{tabular}

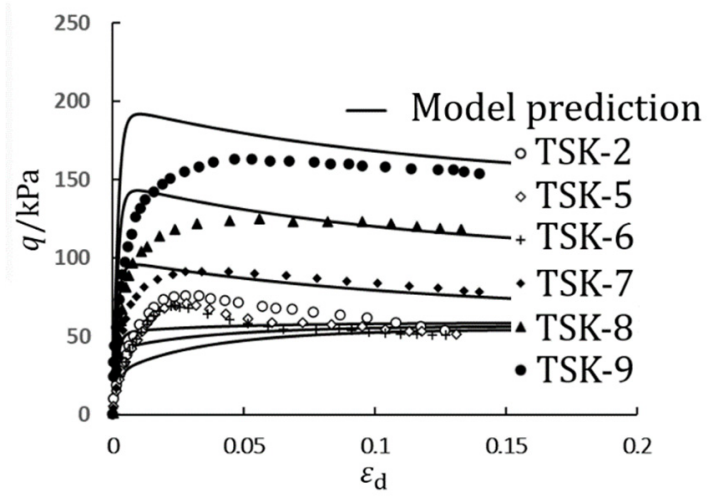

Figure 8. Prediction of undrained shear tests on Osaka clay

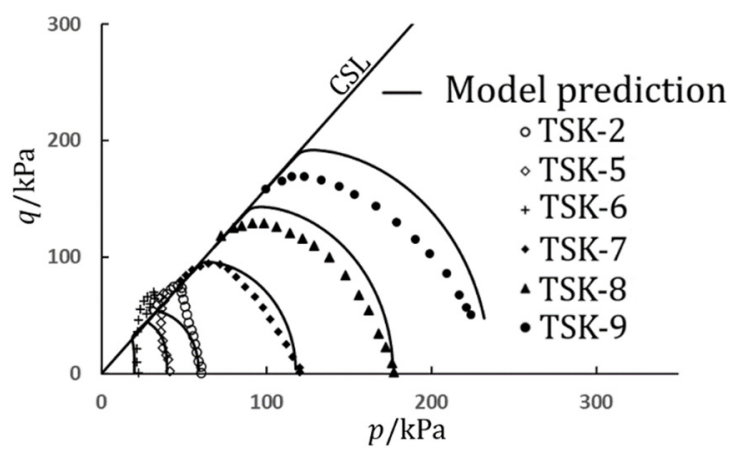

Figure 9. Prediction of effective stress paths of undrained shear tests on Osaka clay

\section{Conclusion}

On the basis of the structured UH model mainly considering soil structure collapse, an EVP structured UH model is extended. In the presented model:

(1) The MNCL in the structured UH model is extended to a MINCL, and the plastic strains due to loading is calculated referring the dynamic MINCL;

(2) A modified description of the internal variable $R_{\mathrm{t}}$ is provided to calculate the plastic strains due to time effect;

From the comparisons between the test data and the corresponding model simulations, the presented EVP structured UH model is able to simply take into account of both the soil structure and the time effect.

\section{Acknowledgement}

This work was supported by the National Natural Science Foundation of China (Grant No. 51809003).

\section{References}

1. He K. S., Shen Z. J., Elasto-viscoplastic damage model for structural clays. Hydro-Science and Engineering, 12(4), 7-13 (2002).

2. Chen T. L., Li. Y. G., Shen. Z. J, Viscous constitutive model for structured clays, Hydro-Science and Engineering, 6(2), 7-11 (2003).

3. Yin Z. Y., Elastic viscoplastic models for natural soft clay: review and development, Chinese Journal of Geotechnical Engineering, 33(9), 1358-1369, (2011).

4. Yin Z. Y., Wang J. H., A one-dimensional strain-rate based model for soft structured clays, Science China, Technological Sciences, 55(1), 90-100 (2012).

5. Zeng L. L., Liu S. Y., Hong Z. S., Modified EVP model considering effect of soil structure, Journal of Southeast University (natural science edition), 42(2), 346-351(2012).

6. Ke W. H., Chen J., Sheng Q., One-dimensional 
elasto-viscoplastic model for structured soft clays, Chinese Journal of Geotechnical Engineering, 38(3), 494-502 (2016).

7. Zhu E. Y., Yao Y. P., A UH constitutive model for structured soils, Rock and soil Mechanics, 36(11), 3101-3110 (2015).

8. Yao Y. P., Kong L. M., Hu J., An elastic-viscous-plastic model for overconsolidated clays, Sci China Tech Sci, 43(3), 298-314 (2013).

9. Jia R., Consolidation behavior of Ariake clay under constant rate of strain, Saga University, (2012).

10. Leroueil S., Kabbaj M., Tavenas F., et al.,
Stress-strain-strain rate relation for the compressibility of sensitive natural clays, Géotechnique, 35(2), 159-180 (1985).

11. Leroueil S., Kabbaj M., Tavenas F., Study of the validity of a $\sigma_{\mathrm{v}}^{\prime}-\varepsilon_{\mathrm{v}}-\dot{\varepsilon}_{\mathrm{v}}$ model in in-situ conditions, Soils and Foundations, 28(3), 13-25 (1988).

12. Adachi T., Oka F., Hirata T., et al. Stress-strain behavior and yielding characteristics of eastern Osaka clay, Journal of the Japanese Geotechnical Society Soils \& Foundation, 35(3), 1-13 (1995). 